\title{
DWARF ELLIPTICAL GALAXIES IN THE M81 GROUP: THE STRUCTURE AND STELLAR POPULATIONS OF BK5N AND F8D1
}

\author{
TAFT E. ARMANDROFF \\ Kitt Peak National Observatory, National Optical Astronomy Observatories, \\ P.O. Box 26732, Tucson, Arizona 85726, U.S.A. \\ NELSON CALDWELL \\ F. L. Whipple Observatory, Smithsonian Institution, P.O. Box 97, \\ Amado, Arizona 85645, U.S.A. \\ G. S. DA COSTA \\ Mount Stromlo and Siding Spring Observatories, The Australian National University, \\ Private Bag, Weston Post Office, ACT 2611, Australia \\ AND \\ PATRICK SEITZER \\ Department of Astronomy, University of Michigan, \\ Ann Arbor, Michigan 48109, U.S.A.
}

\section{Discussion}

The M81 group is one of the nearest groups of galaxies, but its properties are quite different from those of the Local Group. It has therefore provided a different environment for the evolution of its member galaxies. We have carried out a CCD survey of the M81 group to search for analogs to Local Group dwarf elliptical (dE) galaxies. All the M81 dwarfs previously identified in photographic surveys were recovered and we also discovered several new systems whose surface brightnesses fall within the range found for Local Group dE's.

We have obtained HST WFPC2 images through the F555W and F814W filters of two M81 group dE's: BK5N and a new system, designated F8D1. The resulting color-magnitude diagrams show the upper two magnitudes of the red giant branch. The I magnitudes of the red giant branch tip in both galaxies yield distances that are consistent with membership in the M81 group. Surface brightness and total magnitude measurements indicate that BK5N and F8D1 have similar central surface brightness (24.5 and $25.4 \mathrm{mag} \operatorname{arcsec}^{-2}$ in $\mathrm{V}$, respectively), but F8D1's larger length scale results in it being 3 magnitudes more luminous than BK5N. BK5N lies on the relation between central surface brightness and absolute magnitude defined by Local Group dwarf ellipticals, but F8D1 does not. F8D1 is more luminous for its central surface brightness than the relation predicts, similar to the large low surface brightness dwarf galaxies found in, for example, Virgo. The mean color of the giant branch is used to establish the mean abundance of each galaxy. F8D1, the more luminous galaxy, is significantly more metal rich $([\mathrm{Fe} / \mathrm{H}] \approx-1.0)$ than BK5N $([\mathrm{Fe} / \mathrm{H}] \approx-1.7)$. Both BK5N and F8D1 lie on the relation between absolute magnitude and metal abundance defined by Local Group dwarf ellipticals. However, as regards the relation between central surface brightness and metal abundance, BK5N again follows the Local Group dwarfs, while F8D1 deviates significantly from this relation. This suggests that the total amount of luminous matter is more fundamental in controlling metal enrichment than the surface density of luminous matter. We have also used the color width of the giant branch compared with the photometric errors to establish abundance ranges in both galaxies, the sizes of which are comparable to those in Local Group dE's.

From the numbers and luminosities of asymptotic giant branch stars more luminous than the red giant branch tip, we infer that, again like many of the Local Group dE's, both BK5N and F8D1 have had extended epochs of star formation. F8D1 contains stars as young as 3-4 Gyr, while in BK5N stars as young as $\sim 8$ Gyr are present. The fractions of intermediate-age population, at 
$\sim 30 \%$, are similar in both galaxies. Further, one globular cluster has been found in F8D1, but none are present in BK5N. These numbers of clusters are consistent with our expectations from Local Group dwarfs.

Overall, we find that BK5N is similar in all respects to the Local Group dE's. Thus, in spite of the different environments of the M81 group and the Local Group, dwarf galaxies of very similar properties are present in each group. F8D1, on the other hand, has no Local Group counterpart and is indeed rare even in large clusters of galaxies. Our study of its stellar population is the first for a large low surface brightness dwarf.

The full text of this paper is scheduled for publication in the February 1998 issue of the Astronomical Journal. 